\title{
An Overview of Service Models for Urgent and Emergency Psychiatric Care
}

\section{Abstract}

There is significant variation in the way mental health services respond to urgent and emergency presentations, with few evidence based models reported in the literature, and no agreed upon 'best practice' models. To inform the development of urgent and emergency psychiatric care models, a literature review was conducted. The review sought to identify strengths and critique of varying models, evidence gaps, and area for future research. The review process was informed by narrative scoping review methodologies. Relevant studies were identified by searching a range of databases and reference lists. Only article pertaining services that provide rapid access to face to face assessment and/or treatment care (within 24 hours of referral) were included for full review. Thematic analysis of article content was conducted, with the results reported narratively. There is significant variation in the design and scope of urgent and emergency care models. Most models are either community or hospital based, with a few examples of integrated models that span community and hospital care. The development of integrated models has the potential to reduce service duplication and support a shift towards the provision of least restrictive care. The overall evidence base of urgent and emergency care models is limited with few studies in this space, and there is a need for further research. 


\section{Introduction}

A mental health crisis or psychiatric emergency occurs when an individual is faced with an experience that exceeds their ability to cope (Allen, Forster, Zealberg, \& Currier, 2002; Peterman \& Nijman, 2011). When a person experiences a mental health crisis, there is a need, or perceived need, for an urgent or immediate response (Paton, Wright, \& Ayre, 2016; Peterman \& Nijman, 2011; Sunderji, Tan de Bebiana, \& Stergiopoulos, 2015). Persons experiencing a mental health crisis are unable to safely await routine community care, for example owing to the risk of suicide, self-harm or clinical deterioration (Murphy, Irving, Adams, \& Driver, 2012; Sunderji et al., 2015). People in crisis have a number of service options, and can generally access urgent or emergency psychiatric care by presenting to a public hospital Emergency Department (ED) or being referred to an acute community public mental health team. Crisis oriented services provide rapid access to mental health assessment and/or short term treatment in a community or outpatient setting for patients with acute mental health needs.

The provision of urgent and emergency care for people in mental health crisis is an issue of considerable interest, in Australia and globally (Commonwealth of Australia, 2009; Department of Health and Ageing, 2013; Mental Health Commission, 2014a, 2014b; Mental Health Taskforce, 2016; NSW Health, 2017). There is an expectation that health services develop and implement high quality models of care that respond to patient needs in a timely, safe and sensitive manner. With the advent of evidence based practice, service managers and clinicians are expected to engage with the academic literature to inform service development and improvement initiatives. This can be a daunting task for clinicians, especially when the available literature is vast and unwieldy, and when the reported service models/interventions are poorly defined, as is the case here. There is significant variation in the way in which mental health services provide urgent and emergency care, with few 
evidence based models reported in the literature, and no agreed upon 'best practice' models (Paton et al., 2016; Simakhodskaya, Haddad, \& Quintero, 2009; Sunderji et al., 2015). Furthermore, there is a lack of consistent definition and nomenclature, with terms such as 'acute', 'crisis', 'urgent' and 'emergency' care often used interchangeably in reference to urgent and emergency psychiatric services that appear similar in scope.

The aim of the current paper is to provide an overview of models of urgent and emergency mental health care that is accessible to clinicians and service managers to inform service design and improvement initiatives. The purpose of this paper is not to review all papers published but rather to map this complex and heterogeneous field, generate recommendations for the design and delivery of crisis-oriented psychiatric services and identify directions for future research.

\section{Method}

A literature review was conducted to identify current service models that provide an urgent or emergency response to people in mental health crisis, the strengths and critiques of the varying models, and directions for future research. As the aim of the review was to provide an overview of the field, not to provide a comprehensive systematic review, the review process was iterative and informed by narrative (Cronin, Ryan, \& Coughan, 2008; Ferrari, 2015) and scoping review methodologies (Peters et al., 2015). As per the scoping review methodology, quantitative and qualitative evidence were systematically considered to answer a broad research question (Arksey \& O’Malley, 2005; Davis, Drey, \& Gould, 2009; Levac, Colquhoun, \& O'Brien, 2010; Peters et al., 2015). As per the narrative approach, results are displayed descriptively rather than graphically or tabularly as favoured in scoping studies (Arksey \& O’Malley, 2005; Peters et al., 2015). 
Relevant studies were identified through a range a methods. In first instance, the databases Medline, PsychINFO, CINAHL, Embase, and EBM Reviews (including the Cochrane Library) and PubMed were searched for the past 15 years (2002 onwards) using the key words such as 'psychiatric emergency care', 'urgent psychiatric care', 'mental health crisis care' 'acute mental health care' and 'psychiatric crisis care'. Following this, the reference lists of articles were examined for further suitable studies, including seminal and influential works regardless of their publication date.

All articles were screened by the author by reading the abstract and intervention/service description. Articles that described an urgent or emergency psychiatric care service - defined as a service that provides rapid access to a face-face assessment and/or short term treatment for people in mental health crisis, with a response of within 24 hours from referral or presentation - were included for full review. Articles that did not describe a model, or described a model that did not meet the definitional requirements, were excluded. Empirical as well as theoretical articles were included, regardless of approach or study design used.

Information relevant to the research question (i.e. model description; study design; outcomes/results) was extracted from each article. Thematic analysis of the extracted information was conducted to identify different service models, their strengths and limitations, recommendations for service design and areas for future research. The results are reported narratively.

\section{Findings}

Service models that provide urgent and emergency care are varied and include acute community based services, hospital based services in the ED, and to a lesser extent models that integrate community and hospital based acute care (Kowal, Swenson, \& Aubry, 2011; Sunderji et al., 2015; Wand, D'Abrew, Acret, \& White, 2016). 


\section{Acute or Crisis Psychiatric Care in the Community}

There are three main crisis community models, although these models are often interconnected: 1) acute/crisis assertive outreach models; 2) community crisis clinics; and 3) joint crisis models between mental health and police and/or ambulance.

\section{Acute or Crisis Outreach Models}

Services that provide urgent or emergency outreach in the community are commonly described as crisis response and acute care services. Such services are generally similar in scope, and provide rapid access to assessment ( $<24$ hours), referral and/or short term treatment (of up to three to four weeks) to high risk psychiatric groups, specifically people in mental health crisis and those requiring acute follow post a mental health inpatient admission (Althaus et al., 2011; Lloyd-Evans et al., 2017; Murphy et al., 2012; National Institute for Mental Health, 2006; Vigod et al., 2013). Care is generally provided in the least restrictive environment, most commonly a consumer's home or a community centre, by a mental health nurses or a multidisciplinary team. The primary aim of such services is to prevent a crisis or acute situation from worsening and becoming an emergency and leading to an inpatient (re)admission (Chisholm \& Ford, 2004; Lloyd-Evans et al., 2017; Murphy et al., 2012; National Institute for Mental Health, 2006; Sunderji et al., 2015).

While there is little empirical evidence around which exact components of acute community care are critical or essential (Wheeler, Lloyd-Evans, \& Churchard, 2015), evidence indicates that community based model of crisis care are more acceptable to consumers and as clinically effective as inpatient care, with no increased risk (Chisholm \& Ford, 2004; Hasselberg, Grawe, Johnson, \& Ruud, 2011; Murphy et al., 2012; NSW Health, 2008). For 
people who do not require immediate intervention ( $<2$ hours), acute community care provides a number of benefits over hospital based services. They are better able to connect patients to ongoing care as appropriate in the community; provide enhanced patient and carer experience and are more cost-effective (Lloyd-Evans et al., 2017; Murphy et al., 2012; Paton et al., 2016; Sunderji et al., 2015). Further research is required to identify which components of care lead to positive outcomes, to inform the development of an evidence based model that can be consistently implemented (Wheeler et al., 2015).

\section{Community Crisis Clinics}

In addition to outreach services, some acute community models also encompass crisis clinics or crisis day treatment in an effort to meet the need for immediate access and high intensity (Heyland, Emery, \& Shatttell, 2013; Naylor, Taggart, \& Charles, 2017; Paton et al., 2016; Simakhodskaya et al., 2009). Crisis clinics provide safe places for people in crisis to attend until their levels of distress have reduced and become manageable (Agar-Jacomb, 2009; Heyland et al., 2013; Shattell et al., 2014).

There are few community crisis clinics reported in the literature, with the exception of the nurse run clinic 'The Living Room' described by Heyland et al. (2013). An evaluation of this model indicates that providing a safe space for people in crisis to attend is effective in reducing levels of distress, so people can go home having avoided an ED presentation and potential hospital admission (Heyland et al., 2013; Shattell et al., 2014). Community crisis centres can be hard to establish and tend to have limited hours of operation and relatively low uptake (only reducing ED presentations minimally) (Heyland et al., 2013; Simakhodskaya et al., 2009). Despite efforts to manage people in the community, many people in crisis continue to present to the ED and the culture shift required to redirect people in crisis to a community alternative is significant (Heyland et al., 2013; Simakhodskaya et al., 
2009). Further research is required to identify the effectiveness, including cost effectiveness of crisis clinics, and barriers and enablers to their implementation.

Joint crisis models between mental health and police and/or ambulance

Police and ambulance play an important role in responding to people in mental health crisis. Police and ambulance often transport people in mental health crisis to EDs for an assessment and/or treatment. Estimates indicate that around twenty percent of all mental health presentations to EDs are brought in by police (S. Lee, Brunero, \& Fairbrother, 2008).

To reduce transportation to hospital and provide more immediate care in the community, increasingly services are being established conjointly between mental health and police and/or ambulance (Allen Consulting Group, 2012; Boscarato et al., 2014; Forchuk, Jensen, \& Martin, 2010; Herrington, 2012; Hollander, Lee, \& Tahtalian, 2012; Huppert \& Griffiths, 2015; Jones, 2017; Kisely, Campbell, \& Peddle, 2010; S. Lee et al., 2015; McKenna, Furness, \& Oakes, 2015; Naylor et al., 2017). Evaluations of such models indicate that interagency collaborations between mental health nurses and police and/or ambulance improve patient and system outcomes, such as reduction hospitalisation and cost effectiveness (Allen Consulting Group, 2012; S. Lee et al., 2015; Naylor et al., 2017; Paton et al., 2016; Scott, 2000).

\section{Psychiatric Care Models in the Emergency Department}

People presenting to EDs in crisis seeking mental health care compromise between four and ten percent of all presentations (D. Clarke, Dusaome, \& Hughes, 2007; S. Lee et al., 2008; S. Lee et al., 2015; Tankel, Di Palma, Kramer, \& van der Zwan, 2011). The effective management of this population is an issue of considerable interest. Mental health 
presentations tend to cause issues around patient flow in ED; they generally take much longer to assess and ED staff commonly report not feeling equipped to treat this patient group (D. Clarke et al., 2007; Frank, Fawcet, \& Emmerson, 2005; Knott, Pleban, Taylor, \& Castle, 2007).

Mental health services are expected to implement models of care that streamline transition through the ED (Frank et al., 2005; Wand et al., 2016), however, there is significant variation in the way in which mental health care is provided in ED, with no best-practice model. Emergency psychiatric care tends to be provided either in the general ED, through in-reach from specialist mental health nurses or in a designated area or unit co-located with ED specific for psychiatric patients in crisis. The former encompasses models such as Consultation Liaison (CL) or the Emergency Department Mental Health model (EDMH); the latter includes services such as Emergency Psychiatric Assessment and Observation Centres or Psychiatric Emergency Centres (PECs).

\section{Consultation Liaison/ Emergency Department Mental Health models}

In EDs that do not operate a separate psychiatric emergency unit, specialist mental health care is provided by the CL or EDMT team within the general ED. In CL models assessments are conducted by clinicians, predominantly mental health nurses, associated with the community assessment team or hospital psychiatry CL service (eg.Sands, 2007); in EDMH models mental health nurses are based in the ED (24/7) (e.g. Wand, 2016). The CL model works well in small hospitals and is not expensive (Frank et al., 2005; Knott et al., 2007). The EDMH model is more costly than the CL model and more appropriate for medium to large hospital (Frank et al., 2005).

These models are generally not popular with ED staff who often experience long delays before a person is assessed or accepted by the mental health service (Frank et al., 2005; 
Knott et al., 2007). In these models, the mental health service does not take early responsibility for the patient, and generally, if there is no bed, the patient remains the responsibility of the ED.

Furthermore, CL or EDMH models do not address consumer concerns about the appropriateness of the ED environment for mental health patients reported in the literature. Concerns reported by consumers around the suitability of the ED environment include extended waiting times in an uncomfortable environment while in distress; lack of privacy; overstimulating and frightening environment (e.g. crowded, loud); poor access to facilities and services; negative attitudes of ED treatment staff; and tendency for assessments to be repeated (D. Clarke et al., 2007; Heyland et al., 2013; Mental Health Council of Australia, 2005; Shattell \& Andes, 2011; Shattell et al., 2014; Wand, D'Abrew, Barnett, Acret, \& White, 2015).

Despite these criticisms, Wand et al. (2016) promotes an EDMH model that is nurse practioner lead, based in ED 24/7, as 'best practice' arguing that this is the emergency model of choice (Wand et al., 2016; Wand et al., 2015). Despite overall positive feedback, an evaluation of this model identified patient flow as an issue, with patients left waiting in ED for a mental health bed to become available (Wand et al., 2016; Wand et al., 2015). While $63 \%$ of patients were seen within less than two hours, nearly ten percent of patients waited more than ten hours (Wand et al., 2015).

\section{Psychiatric Emergency Centres}

ED Psychiatric Assessment and Observation Centres, often called Psychiatric Emergency Centres (PECs) have a long history in the United States (Breslow, Klinger, \& Erickson, 1995; P. Clarke, Hafner, \& Holme, 1997; Currier \& Allen, 2003; Oldham \& DeMasi, 1995), and more recently Australia (Australasian Health Facility Guidelines, 2010; Frank et al., 2005; 
Garling, 2008; JA Projects, 2012; Ministry of Health, 2015). PECs are assessment and short term treatment and observation units for people who present to ED in mental health crisis (Brakoulias, Mandali, Seymour, Sammut, \& Starcevic, 2010; Frank et al., 2005; JA Projects, 2012; Simakhodskaya et al., 2009). Such units are costly to run compared to the CL and EDMH models, and are generally recommended for larger hospitals (Currier \& Allen, 2003; Frank et al., 2005).

PECs are usually located adjacent to EDs, and operate as an extension of the mental health triage and assessment service in the ED. To support patient flow, patients identified as requiring a specialist mental health assessment and/or observation can be fast tracked into PEC (Australasian Health Facility Guidelines, 2010; Frank et al., 2005; JA Projects, 2012; Kalucy, Thomas, \& King, 2005). PECs are designed to improve the ED environment in which people in mental health crisis are assessed and treated, by providing a less stimulating, less rushed and more sensitive environment (Frank et al., 2005).

The less rushed environment of PECs compared to general EDs allows for increased time to gain diagnostic clarity (avoid misdiagnosis) and develop alternatives to hospitalisation (Allen et al., 2002; Breslow et al., 1995; T. Lee, Renaud, \& Hills, 2003). Patients can be observed for a time-limited period, potentially avoiding a conventional psychiatric inpatient admission (Frank et al., 2005). Many psychiatric crises are brief and resolve spontaneously (Allen et al., 2002; Breslow et al., 1995; Breslow, Klinger, \& Erickson, 1996; National Health and Medical Research Council, 2013); while some crisis cannot be managed in the community, they can potentially be managed in PECs averting longer term admissions (Allen et al., 2002; Breslow et al., 1995; Chisholm \& Ford, 2004; Gillig et al., 1989; lanzito, Fine, Sprague, \& Pestana, 1978; Oldham \& DeMasi, 1995).

PECs also allow for better monitoring of patients with reduced risk of self-harm or absconding (Frank et al., 2005). Instead of waiting in the ED waiting area, patients requiring 
a mental health assessment can be transferred to PEC. PECs provide a comfortable and monitored environment in which patients can wait comfortably, likely reducing the number of patients who leave the ED before the assessment has been completed. Focus group evidence from D. Clarke et al. (2007) indicates that from the perspective of consumers and carers being transferred to a mental health area that is appropriately staffed and monitored is a successful outcome of an ED visit. This study found that people that present to ED in crisis want to be taken seriously, and not abandoned in a waiting room or rushed out the door which they report often occurs (D. Clarke et al., 2007).

While PECs have the potential to meet a range of patient and system needs, they have not been rigorously evaluated, and their effectiveness remains largely unclear. Limited evidence comes from an evaluation of 13 PECs in New South Wales, Australia conducted in 2012 (JA Projects, 2012). This evaluation found that while PECs are viewed positively by consumers, carers and clinicians, they had not been implemented as intended. The majority of PECs had not been implemented as assessment and observation units, but rather as short stay mental health units for vulnerable populations (JA Projects, 2012). Patients were not fast-tracked into PEC for assessment, and as such, the introduction of PECs had little impact on patient flow in EDs (JA Projects, 2012). The reported reasons for this included issues around assessment space in the PECs, lack of medical in-reach from ED into PEC for the management of physical comorbidities, and difficulties transferring patients back to ED if required (JA Projects, 2012). Given poor implementation fidelity, this evaluation does not offer significant insight into the effectiveness of PECs.

Even though PECs have not been comprehensively studied and their effectiveness remains unclear, the model has received some criticism, with some scholars arguing that PECs provide a segregated system of health care that is discriminatory to mental health patients (Wand et al., 2016; Wand \& White, 2007). These scholars argue that mental health patients prefer mental health care to be provided in the same physical environment as patients with 
physical health emergencies (Wand \& White, 2007). This argument is not well supported as consumer preferences in the way in which psychiatric care is delivered is not well established. While a study by D. Clarke et al. (2007) found that some people with mental illness who participated in a focus group did not want a separate psychiatric emergency, a survey study by Summers and Happell (2003) found the opposite. Further research into the perspective of consumers in this space is required.

\section{Towards the development and delivery of integrated service}

\section{models}

The way in which crisis oriented services operate varies significantly, in particular around service scope and boundaries. A common way to delineate service boundaries is by separating responsibility for urgent versus emergency care, with urgent care provided by acute community teams and emergency care by mental health clinicians in the ED. This is fraud with difficulties; people in crisis can transition between requiring urgent versus emergency care rapidly, they commonly present to EDs for non-emergency problems, and emergencies can often be managed effectively in the community (Allen et al., 2002; Althaus et al., 2011; Heyland et al., 2013; Southern, Leahey, Harper-Jaques, McGonigal, \& Syverson, 2007; Sunderji et al., 2015; Vandyk, Harrison, VanDenKerkhof, Graham, \& RossWhite, 2013).

There is a push for flexible service models to be developed that reduce traditional artificial service boundaries by integrating urgent, emergency, community and hospital based care (Mental Health Taskforce, 2016; Naylor et al., 2017). Artificial boundaries lead to fragmented and inefficient service system that are rich in duplication and treat different components of care in isolation (Mental Health Taskforce, 2016; Naylor et al., 2017; Southern et al., 2007). The separation between hospital and acute community care is a prime example of an 
artificial boundary, and patients accessing hospital services usually lose access to community expertise and resources (Allen et al., 2002; Mental Health Taskforce, 2016; Naylor et al., 2017). There is a need to develop services that integrate community and hospital care to deliver services in the least restrictive environment appropriate for the consumer. The development of services that support a shift from inpatient treatment to community management is a national and international mental health reform priority (Commonwealth of Australia, 2009; Department of Health and Ageing, 2013; Mental Health Commission, 2014a, 2014b; Mental Health Taskforce, 2016; NSW Health, 2017).

One strategy commonly suggested to improve integration between community and hospital based services is the assessment of all patients potentially requiring an inpatient admission by a central acute community team (Begum \& Riordan, 2016; Chisholm \& Ford, 2004; Hasselberg et al., 2011; Murphy et al., 2012; National Institute for Mental Health, 2006). Community emergency assessment by one central team can help ensure inpatient care is used appropriately and only when necessary (least restrictive care is provided), and in turn, reduce admissions and pressure and beds (Chisholm \& Ford, 2004; Hasselberg et al., 2011; Hugo, Smout, \& Bannister, 2002; Murphy et al., 2012; National Institute for Mental Health, 2006). A study by Hugo et al. (2002) compared the rates of inpatient admission between a community-based psychiatric emergency service and a hospital-based psychiatric emergency service found that people assessed by a community team are three times less likely to be admitted, regardless of clinical characteristics. These authors call for psychiatric emergency services to include a community focussed mobile assessment team to provide services in the least restrictive environment and help avoid hospitalisation.

PECs can also play a role in integrating acute community, emergency and inpatient care. While hospital based, the focus of PECs is on the resources available to the patient outside the hospital, in the community (Allen et al., 2002; Frank et al., 2005; Simakhodskaya et al., 2009). Similar to crisis clinics in the community, by providing a safe crisis observation space, 
PECs have the potential to reduce hospitalisation and support people in psychiatric crisis to remain in the community (Paton et al., 2016; Simakhodskaya et al., 2009). Similar to crisis clinics or day centres, PECs can provide a safe place for people in crisis to attend until their levels of distress have reduced and become manageable (Paton et al., 2016). There is often uncertainty and indecisiveness around the most appropriate care pathway for people in crisis, and capacity for observation can reduce avoidable admissions. In addition, the less rushed environment of PECs offers the ability to provide interventions at the time of crisis presentation, allowing for this critical point to be used as an opportunity for intervention (Simakhodskaya et al., 2009).

PECs can be implemented as a component of an integrated model that encompasses urgent and emergency crisis care in the community and hospital (Frank et al., 2005; Simakhodskaya et al., 2009). An example of this is the model described by Simakhodskaya et al. (2009), which encompasses a range of integrated psychiatric emergency crisis services. This integrated model includes emergency assessments in the ED, an extended assessment and observation unit (co-located with ED) and crisis community care. Similarly, the PEC model described by Frank et al. (2005) also encompasses these key components, and has capacity for emergency assessment, crisis intervention and observation, as well as strong integration with acute community teams. This PEC is managed within a community model, to support a shift away from inpatient management.

\section{Implications for practice}

This review identified five main urgent and emergency psychiatric care models across community and hospital settings. While similar in scope in the sense that each model provides urgent or emergency care, the models complement each other and have unique (while overlapping) service criteria. Acute/crisis assertive outreach models respond to people in the community; community crisis clinics provide a 'safe' place to people in crisis; joint 
crisis models between mental health and police and/or ambulance provide care to people that require police and ambulance involvement; PECs and EDMH and CL models provide specialist mental health care for people in ED (either in PEC, or for those patients that are better managed in ED (e.g. those with comorbid medical issues) through in-reach by an $\mathrm{EDMH} / \mathrm{CL}$ service). While none of these models can operate in isolation, as together they cover the spectrum of urgent and emergency psychiatric care, it is suggested here that these models could be better integrated and delivered as one service, potentially by one integrated multidisciplinary team. It is suggested that improved integration can lead to positive system and patient outcomes, by reducing service duplication and fragmentation.

\section{Directions for Future Research}

There is a need for further research into the provision of urgent and emergency psychiatric care, including studies that identify implementation barriers and assess program fidelity as well as high quality studies that compare different models in terms of consumer outcomes and perspectives. There is overall little consistency in the way models are designed and implemented, with poor program fidelity. While there is evidence to support the effectiveness of acute/crisis community care, there is significant variation in the way these models operate and it remains unclear which components of care contribute to positive outcomes. While the importance of developing psychiatric emergency models for people that present to ED in mental health crisis is well recognised, there is significant variation in the way services are implemented, and it remains unclear which models are more effective and acceptable to consumers. Furthermore, there is a need for urgent and emergency care models that are integrated across community and hospital settings to be developed and trialled. Despite a push towards integrated service delivery, little is understood around how integrated urgent and emergency mental health care is best delivered, what the implementation barriers and enablers may be, and how effective and acceptable to consumers integrated models are. 


\section{Conclusion}

Urgent and emergency psychiatric care is a complex area of investigation, with a lack of consistent definition and nomenclature. The overall evidence base of urgent and emergency care models is poor, with few evidence based models reported in the literature. There is significant variation in the design and scope of urgent and emergency care models, with most models either community based or hospital based. The literature review identified a call for the development of service models that integrate urgent and emergency care across community and hospital setting to reduce service duplication, and support a shift towards the provision of least restrictive care. While not necessarily comprehensive owing to heterogeneous nomenclature and the iterative review methodology used, this study provides an overview of urgent and emergency psychiatric care models and recommendations towards the development of integrated service models and directions for future research. 


\section{References}

Agar-Jacomb, K. R., J. . (2009). Mental health crisis services: What do service users need when in crisis? . Journal of Mental Health, 18(2), 99-110.

Allen Consulting Group. (2012). Police, Ambulance and Clinical Early Response (PACER) evaluation: Final report. Melbourne: Report to Department of Health, Victoria.

Allen, M., Forster, P., Zealberg, J., \& Currier, G. (2002). APA Task Force on Psychiatric Emergency Services: Report and Recommendations Regarding Psychiatric Emergency and Crisis Services. A Review and Model Program Description.

Althaus, F., Paroz, S., Hugli, O., Ghali, W., Daeppen, J., Peytremann-Bridevaux, I., \& Bodenmann, P. (2011). Effectiveness of interventions targeting frequent users of emergency departments: A systematic review. Ann Emerg Med, 58(1), 41-52.

Arksey, H., \& O’Malley, L. (2005). Scoping studies: Towards a methodological framework. International Journal of Social Research Methodology: Theory \& Practice, 8(1), 1932.

Australasian Health Facility Guidelines. (2010). Part B - Health Facility Briefing and Planning: 133 psychiatric emergency care centre (PECC). Revision v.4.0. Australasian Health Infrastructure Alliance.

Begum, R., \& Riordan, S. (2016). Nurses experiences of working in Crisis Resolution Home Treatment Teams with its additional gatekeeping responsibilities. Journal of Psychiatric \& Mental Health Nursing.

Boscarato, K., Lee, S., Kroschel, J., Holander, Y., Brennan, A., \& Warren, N. (2014). Consumer experience of formal crisis-response services and preferred methods of crisis intervention. International Journal of Mental Health Nursing, 23(4), 287-295.

Brakoulias, V., Mandali, R., Seymour, J., Sammut, P., \& Starcevic, V. (2010). Characteristics of admission to a recently opened Psychiatric Emergency Care Centre. Australasian Psychiatry, 18(4), 326-329. 
Breslow, R., Klinger, B., \& Erickson, B. (1995). Crisis Hospitalization in a Psychiatric Emergency Service (M. Allen Ed. Vol. 67). San Francisco: Jossey-Bass.

Breslow, R., Klinger, B., \& Erickson, B. (1996). Acute Intoxication and Substance Abuse Among Patients Presenting to a Psychiatric Emergency Service. General hospital psychiatry, 3(18), 183-191.

Chisholm, A., \& Ford, R. (2004). Transforming Mental Health Care: Assertive outreach and crisis resolution in practice: The Sainsbury Centre for Mental Health/National Institute for Mental Health in England.

Clarke, D., Dusaome, D., \& Hughes, L. (2007). Emergency department from the mental health client's perspective. International Journal of Mental Health Nursing, 16, 126131.

Clarke, P., Hafner, J., \& Holme, G. (1997). The brief admission unit in emergency psychiatry. Journal of Clinical psychology, 53(8).

Commonwealth of Australia. (2009). Fourth National Mental Health Plan: An agenda for collaborative government action in mental health 2009-2014. Retrieved from

Cronin, P., Ryan, F., \& Coughan, M. (2008). Undertaking a literature review: a step-by-step approach. British Journal of Nursing, 17(1).

Currier, G., \& Allen, M. (2003). Organistaion and function of academic psychiatry emergency services. General Hospital Psychiatry, 25, 124-129.

Davis, K., Drey, N., \& Gould, D. (2009). What are scoping studies? A review of nursing literature. Int J Nurs Stud, 46, 1386-1400.

Department of Health and Ageing. (2013). National Mental Health Report 2013: tracking progress of mental health reform in Australia 1993-2011. Commonwealth of Australia, Canberra.

Ferrari, R. (2015). Writing narrative style literature reviews. The European Medical Writers Review, 24(4), 230-234.

Forchuk, C., Jensen, E., \& Martin, M. L. (2010). Psychiatric crisis services in three communities. Canadian Journal of Community Mental Health, 29, 73-86. 
Frank, R., Fawcet, L., \& Emmerson, B. (2005). Development of Australia's first psychiatric emergency centre. . Australasian Psychiatry, 13, 266 - 272.

Garling, P. (2008). Final Report of the Special Commission of Inquiry Acute Care Services in NSW Public Hospitals. Retrieved from

Gillig, P., Hillard, J., Bell, J., Combs, H., Martin, C., \& Deddens, J. (1989). The Psychiatric Emergency Service Holding Area: Effect on Utilization of Impatient Resources. American Journal of Psychiatry Quarterly, 146, 369-372.

Hasselberg, N., Grawe, R., Johnson, S., \& Ruud, T. (2011). Treatment and outcomes of Crisis Resolution teams: A prospective, multi-centre study. BMC Psychiatry, 11(183). Herrington, V. (2012). Inter-agency Cooperation and Joined-up Working in Police Responses to Persons with a Mental Illness: Lessons from New South Wales. Policing: A Journal of Policy \& Practice, 6(4), 388-397.

Heyland, M., Emery, M., \& Shatttell, M. (2013). The Living Room, a Community Crisis Respite Program: Offering People in Crisis an Alternative to Emergency Departments. Global Journal of Community Psychology Practice, 4(3), 1-8.

Hollander, Y., Lee, S., \& Tahtalian, S. (2012). Challenges Relating to the Interface Between Crisis Mental Health Clinicians and Police When Engaging with People with a Mental IIIness. Psychiatry, Psychology and Law, 19(3), 402-411. .

Hugo, M., Smout, M., \& Bannister, J. (2002). A comparison in hospitalization rates between a community-based mobile emergency service and a hospital-based emergency service. Aust N Z J Psychiatry, 36(4), 504-508.

Huppert, D., \& Griffiths, M. (2015). Police Mental Health Partnership project: Police Ambulance Crisis Emergency Response (PACER) model development. Australasian Psychiatry, 23(5), 520-523.

Ianzito, B., Fine, J., Sprague, B., \& Pestana, J. (1978). Overnight Admission for Psychiatric Emergencies. Hospital and Community Psychiatry, 29, 728-730.

JA Projects. (2012). External Review of Psychiatric Emergency Care Centres in NSW: Final report to the NSW Ministry of Health. Endorsed by MHDAO: JA Projects. 
Jones, J. (2017). Kerbside home based mental health triage: a person focused. multiorganisational solution for risk, resources and re-trauma. Paper presented at the Mental Health Innovation Showcase, Sydney.

Kalucy, R., Thomas, L., \& King, D. (2005). Changing demand for mental health services in the emergency department of a public hospital. Australian and New Zealand Journal of Psychiatry Quarterly, 39, 74 - 80.

Kisely, S., Campbell, L. A., \& Peddle, S. (2010). A controlled before-and-after evaluation of a mobile crisis partnership between mental health and police services in Nova Scotia. Canadian Journal of Psychiatry, 55, 662-668.

Knott, J., Pleban, A., Taylor, D., \& Castle, D. (2007). Management of mental health patients attending Victorian emergency departments. The Royal Australian and New Zealand College of Psychiatrists, 41, 759-767.

Kowal, J., Swenson, J., \& Aubry, T. (2011). Improving access to acute mental health services in general hospital Journal of Mental Health, 20(1), 5-14.

Lee, S., Brunero, S., \& Fairbrother, G. (2008). Profiling police presentations of mental health consumers to an emergency department. . International Journal of Mental Health Nursing, 17, 311-316.

Lee, S., Thomas, P., Doulis, C., Bowles, D., Henderson, K., Keppich-Arnold, S., . . . Stafrace, S. (2015). Outcomes achieved by and police and clinician perspectives on a joint police officer and mental health clinician mobile response unit. International Journal of Mental Health Nursing, 24, 538-5646.

Lee, T., Renaud, E., \& Hills, O. (2003). Emergency Psychiatry: An Emergency Treatment Huband-Spoke Model for Psychiatric Emergency Services. . Psychiatric Services, 54, $1590-1594$.

Levac, D., Colquhoun, \& O'Brien, K. (2010). Scoping studies: advancing the methodology. Implementation Science, 2010(5), 69. 
Lloyd-Evans, B., Paterson, B., Onyett, S., Brown, E., Istead, H., Gray, R., . . Johnson, S. (2017). National implementation of a mental health service model: A survey of Crisis Resolution Teams in England. International Journal of Mental Health Nursing.

McKenna, B., Furness, T., \& Oakes, J. (2015). Police and mental health clinician partnership in response to mental health crisis: A qualitative study. . International Journal of Mental Health Nursing, 24(5), 386-393.

Mental Health Commission. (2014a). Living Well: A Strategic Plan for Mental Health in NSW. Sydney: NSW Mental Health Comission,.

Mental Health Commission. (2014b). Living Well: Putting people at the centre of mental health reform in NSW. Retrieved from Sydney: http://nswmentalhealthcommission.com.au/node/1586

Mental Health Council of Australia. (2005). Not For Service: Experiences of Injustice and Despair in Mental Health Care in Australia. Canberra.

Mental Health Taskforce. (2016). The five year forward view for Mental Health: A report from the independent Mental Health Taskforce to the NHS in England. Retrieved from https://www.england.nhs.uk/wp-content/uploads/2016/02/Mental-Health-Taskforce$\underline{\text { FYFV-final.pdf }}$

Ministry of Health. (2015). Guideline: Psychiatric Emergency Care Centre Model of Care Guideline. Document Number GL2015_009. Retrieved from North Sydney:

Murphy, S., Irving, C., Adams, C., \& Driver, R. (2012). Crisis intervention for people with severe mental illnesses. Cochrane Database of Systematic Review. Issue 5, Art. No.: CD001087. DOI: 10.1002/14651858.CD001087.pub4.

National Health and Medical Research Council. (2013). Clinical Practice Guideline for the Management of Borderline Personality Disorder. Retrieved from Canberra:

National Institute for Mental Health. (2006). Guidance Statement on Fidelity and Best Practice for Crisis Services: Care Services Improvement Partnership, Department of Health and National Institute for Mental Health in England. 
Naylor, C., Taggart, H., \& Charles, A. (2017). Mental health and new models of care: Lessons from the vanguards. Retrieved from http://www.rcpsych.ac.uk/pdf/MH new models care Kings Fund May 2017.pdf, accessed 17 June 2017

NSW Health. (2008). NSW community mental health strategy 2007-2012: From prevention and early intervention to recovery. North Sydney: NSW Department of Health. NSW Health. (2017). Charter for Mental Health Care in NSW. Retrieved from http://www.health.nsw.gov.au/mentalhealth/Pages/charter.aspx

Oldham, J., \& DeMasi, M. (1995). An Integrated Approach to Emergency Psychiatric Care (M. Allen Ed. Vol. 67). San Francisco: Jossey-Bass.

Paton, F., Wright, K., \& Ayre, N. (2016). Improving outcomes for people in mental health crisis: a rapid synthesis of the evidence for available models of care. Health technology assessment, 1(161).

Peterman, B., \& Nijman, H. (2011). Assessing aggression risks in patients of the ambulatory health crisis team. Community mental Health, 47, 463-471.

Peters, M., Godfrey, C., BPharm, H., Mclnerney, P., Parker, D., \& Soares, C. (2015). Guidance for conducting systematic scoping reviews. International Journal of Evidence-Based Healthcare, 13, 141-146.

Sands, N. (2007). Mental health triage: towards a model of nursing practice. Journal of Psychiatric \& Mental Health Nursing, 14, 243-249.

Scott, R. L. (2000). Evaluation of a mobile crisis program: Effectiveness, efficiency, and consumer satisfaction. Psychiatric Services, 51, 1153-1156.

Shattell, M., \& Andes, M. (2011). Treatment of persons with mental illness and substance use disorders in medical emergency departments in the United States. Issues in Mental Health Nursing, 32(2), 140-141.

Shattell, M., Harris, B., Beavers, J., Tomlinson, K., Prasek, L., Geevarghese, S., . . . Heyland, M. (2014). A Recovery-Oriented Alternative to Hospital Emergency 
Departments for Persons in Emotional Distress: "The Living Room". Issues in Mental Health Nursing, 35(1), 4-12.

Simakhodskaya, Z., Haddad, F., \& Quintero, M. (2009). Innovative use of crisis intervention services with psychiatric emergency room patients. Prim Psychiatry, 16(9), 60-65.

Southern, L., Leahey, M., Harper-Jaques, S., McGonigal, K., \& Syverson, A. (2007). Integrating mental health into urgent care in a community health centre. Can Nurse., 103(1), 29-34.

Summers, M., \& Happell, B. (2003). Patient satisfaction with psychiatry services provided by a Melbourne tertiary hospital emergency department. Journal of Psychiatric \& Mental Health Nursing, 10, 351-357.

Sunderji, N., Tan de Bebiana, J., \& Stergiopoulos, V. (2015). Urgent Psychiatric Services: A Scoping Review. Psychiatry, 60(9), 393-402.

Tankel, A., Di Palma, M., Kramer, K., \& van der Zwan, R. (2011). Increasing impact of mental health presentations on New South Wales public hospital departments 19992006. Emerg Med Australas, 23, 689-696.

Vandyk, A., Harrison, M., VanDenKerkhof, E., Graham, I., \& Ross-White, A. (2013).

Frequent emergency department use by individuals seeking mental healthcare: a systematic search and review. F. Arch Psychiatr Nurs, 27(4), 171-178.

Vigod, S., Kurdyak, K., Dennis, C., Leszcz, T., Taylor, V., Blumberger, D., \& Seitz, D. (2013). Transitional interventions to reduce early psychiatric readmissions in adults: systematic review. British Journal of Psychiatry, 202(3), 187-194.

Wand, T., D'Abrew, N., Acret, L., \& White, K. (2016). Evaluating a new model of nurse-led emergency department mental health care in Australia. International Emergency Nursing, 24, 16-21.

Wand, T., D'Abrew, N., Barnett, C., Acret, L., \& White, K. (2015). Evaluation of a nurse practioner-led extended hours mental health liaison nurse service based in the Emergency Department. Australian Health Review, 39, 1-8. 
Wand, T., \& White, K. (2007). Examining models of mental health service delivery in the emergency department. Aust N Z J Psychiatry, 41, 784-791.

Wheeler, C., Lloyd-Evans, B., \& Churchard, A. (2015). Implementation of the Crisis Resolution Team model in adult mental health settings: A systematic review. BMC Psychiatry, 15(74). 